\title{
Profile of sacubitril/valsartan in the treatment of heart failure: patient selection and perspectives
}

This article was published in the following Dove Press journal:

Vascular Health and Risk Management

5 October 2017

Number of times this article has been viewed

\section{Srikanth Yandrapalli' \\ Gabriela Andries' \\ Medha Biswas ${ }^{2}$ \\ Sahil Khera ${ }^{2,3}$}

'Department of Internal Medicine, New York Medical College at Westchester Medical Center, Valhalla, NY, USA; '2Division of Cardiology, New York Medical College at Westchester Medical Center, Valhalla, NY, USA; ${ }^{3}$ Division of Cardiology, Massachusetts General Hospital, Harvard Medical School, Boston, MA, USA
Correspondence: Sahil Khera Division of Cardiology, Massachusetts General Hospital/Harvard Medical School, 55 Fruit Street, Boston, MA 02114, USA

Tel +l 9145647587

Fax +I 6177267855

Email skhera@mgh.harvard.edu
Abstract: With an estimated prevalence of 5.8 million in the USA and over 23 million people worldwide, heart failure (HF) is growing in epidemic proportions. Despite the use of guidelinedirected medical therapies such as angiotensin-converting enzyme inhibitors, beta-adrenergic blockers, angiotensin receptor blockers, and mineralocorticoid receptor antagonists for chronic systolic HF for almost two decades, HF remains a leading cause of morbidity, mortality, and health care expenditures. The Prospective Comparison of Angiotensin Receptor-Neprilysin Inhibitor with Angiotensin-Converting Enzyme Inhibitor to Determine Impact on Global Mortality and Morbidity in Heart Failure (PARADIGM-HF) trial provided compelling evidence for the cardiovascular and mortality benefit of sacubitril/valsartan when compared to enalapril in patients with heart failure and reduced ejection fraction (HFrEF). Sacubitril/valsartan performed better than enalapril across various HFrEF patient characteristics and showed substantial benefit in patients with other common comorbidities. Following the trial, the US Food and Drug Administration approved this drug for the treatment of HF. Various international HF consensus guidelines endorse sacubitril/valsartan as a class I recommendation for the management of symptomatic HFrEF. Although this high-quality clinical study is the largest and the most globally represented trial in HFrEF patients, concerns have been raised regarding the generalizability of the trial results in real-world HF population. The gaps in US Food and Drug Administration labeling and guideline recommendations might lead to this medication being used in a larger population than it was studied in. In this review, we will discuss the current role of sacubitril/valsartan in the management of HF, concerns related to PARADIGM-HF and answers, shortcomings of this novel drug, effects on patient characteristics, real-world eligibility, and the role of ongoing and further investigations to clarify the profile of sacubitril/valsartan in the management of HF. Keywords: sacubitril/valsartan, Entresto, HFrEF, systolic heart failure, LCZ696, angiotensin receptor neprilysin inhibitor

\section{Introduction}

Heart failure (HF) is associated with significant morbidity, mortality, and health care expenditure. HF is classified based on left ventricular ejection fraction (LVEF) into HF with reduced EF (HFrEF) with an LVEF <40\% and HF with preserved EF (HFpEF) with an LVEF $\geq 50 \%$. ${ }^{1}$ An EF between $40 \%$ and $49 \%$ is considered an intermediate zone and is termed as HF with borderline EF or HF with mid-range EF. Epidemiologic data indicate that HFpEF and HFrEF contribute equally to the total HF population. ${ }^{1}$ HFpEF patients have a similar post-discharge mortality risk and equally high rates of rehospitalization, compared to patients with $\mathrm{HFrEF}^{2}$ With an estimated prevalence of 5.8 million in the USA and over 23 million people worldwide, HF is growing in 
epidemic proportions. ${ }^{3}$ The cost of HF in the USA was around $\$ 30$ billion in 2012, a number that is projected to increase to around $\$ 70$ billion by the year $2030 .^{4}$

Acute decompensated HF (ADHF) is the clinical syndrome of new onset or worsening HF symptoms and signs requiring urgent treatment. ${ }^{5}$ In the USA, ADHF exacerbations result in around one million hospitalizations yearly and contribute largely to the overall HF health care expenditure. ${ }^{4}$ Hospitalization for ADHF serves as a poor prognostic indicator with $\sim 30 \%$ and 50\% readmission rates at 1 and 6 months, respectively, and a 1-year all-cause mortality as high as $30 \%{ }^{6,7}$ The estimated survival rate after the diagnosis of HF is $50 \%$ at 5 years and $10 \%$ at 10 years. ${ }^{8}$ Despite the use of guideline-directed medical therapies such as angiotensinconverting enzyme inhibitors (ACEIs), beta-adrenergic blockers, angiotensin receptor blockers (ARBs), and mineralocorticoid receptor antagonists (MRAs) as cornerstone medical therapies for chronic systolic HF for almost two decades, HF remains a leading cause of morbidity, mortality, and health care expenditures in the USA and worldwide.

Advances in our understanding of the renin-angiotensinaldosterone (RAAS) pathway and natriuretic peptide system, lessons learned from randomized trials of natriuretic peptide system augmentation, and pharmaco-innovation led to the creation and validation of combination sacubitril/valsartan (Entresto $^{\text {TM }}$ [LCZ696]; Novartis) for the treatment of HFrEF. The Prospective Comparison of Angiotensin ReceptorNeprilysin Inhibitor with Angiotensin-Converting Enzyme Inhibitor to Determine Impact on Global Mortality and Morbidity in Heart Failure (PARADIGM-HF) trial provided compelling evidence for the cardiovascular $(\mathrm{CV})$ and mortality benefit of sacubitril/valsartan when compared to enalapril (an ACEI) in patients with HFrEF. ${ }^{9}$ Numerous post hoc analyses of the original trial extended the benefits of this innovative medication across a multitude of clinical characteristics. ${ }^{10}$ Following the trial, the US Food and Drug Administration (FDA) approved this drug for the treatment of HF. International HF consensus guidelines now endorse sacubitril/valsartan as a class I recommendation for the management of HFrEF. ${ }^{11-13}$ In this review, we will discuss the current role of sacubitril/ valsartan in the management of $\mathrm{HF}$, shortcomings of this novel drug, effects on patient characteristics, real-world eligibility, and the role of ongoing and further investigations to clarify the profile of sacubitril/valsartan in the management of HF.

\section{The PARADIGM-HF trial}

LCZ696 is a novel, orally active, first-in-class angiotensin receptor-neprilysin inhibitor (ARNI), which is a $1: 1$ combination of valsartan (an ARB) and the neprilysin inhibitor (NEPI) sacubitril. ${ }^{14}$ Sacubitril (AHU377) is a prodrug, which upon ingestion is rapidly metabolized to an active NEPI moiety LBQ657. ${ }^{14}$ The mechanism of action and the effects of LCZ696 on the CV system in HF are explained in Figure $1 .{ }^{15}$ Preclinical trials of this combination provided evidence for simultaneous neprilysin inhibition and angiotensin receptor blockade. ${ }^{10}$ Favorable safety and efficacy data of this combination from a Phase III randomized controlled trial in hypertensive HFpEF patients paved the way for the larger PARADIGM-HF trial. ${ }^{9}$

The PARADIGM-HF was a Phase III double-blind controlled trial that randomized 8,442 patients with New York Heart Association (NYHA) class II-IV HF and an LVEF of $\leq 40 \%$ (later amended to $\leq 35 \%$ ) to receive twice daily dosing of either $200 \mathrm{mg}$ of LCZ696 or $10 \mathrm{mg}$ of enalapril in addition to standard medical therapy for HF (Table 1). ${ }^{9}$ Patients were eligible for the study if they had elevated plasma B-type natriuretic peptide (BNP) level $\geq 150 \mathrm{pg} / \mathrm{mL}$ (or $\geq 100 \mathrm{pg} / \mathrm{mL}$ if hospitalized for HF in previous 12 months) or elevated N-terminal pro-BNP (NT-proBNP) level $\geq 600 \mathrm{pg} / \mathrm{mL}$ (or $\geq 400 \mathrm{pg} / \mathrm{mL}$ if hospitalized for HF in previous 12 months). At 27-month follow-up, LCZ696 was associated with a 20\% decrease in the composite primary endpoint of death from CV causes or hospitalization for HF, compared to enalapril. Additionally, LCZ696 reduced death from any CV cause by $20 \%$, first hospitalization for worsening HF by $21 \%$, all-cause mortality by $16 \%$, and improved the symptoms and quality of life as measured on the Kansas City Cardiomyopathy Questionnaire (Table 1). ${ }^{9,16}$ Numerous post hoc analyses have demonstrated the superiority of sacubitril/valsartan over enalapril, irrespective of age, systolic blood pressure (SBP), LVEF, baseline HF risk scores, and glycemic status. ${ }^{10}$

LCZ696 exhibited additional clinical benefit over enalapril in HF patients by attenuating progression of the HF disease process. Fewer LCZ696-treated patients required intensification of medical treatment for HF or an emergency department visit for worsening HF. ${ }^{17}$ Patients in the LCZ696 group had 23\% fewer hospitalizations for worsening HF evident within the first 30 days of randomization and were less likely to have implantation of an HF device or cardiac transplantation. ${ }^{17}$ Patients taking sacubitril/valsartan had early and sustained reduction in biomarkers of myocardial wall stress and injury (NT-proBNP and troponin) and increased plasma BNP levels versus enalapril. ${ }^{17}$ Putative placebo analysis of LCZ696 using results from previous landmark placebo-controlled trials of RAAS blockade in $\mathrm{HF}$ showed significantly greater reductions in $\mathrm{CV}$ death, $\mathrm{HF}$ hospitalization, and all-cause mortality with LCZ696 ${ }^{18}$ Findings 


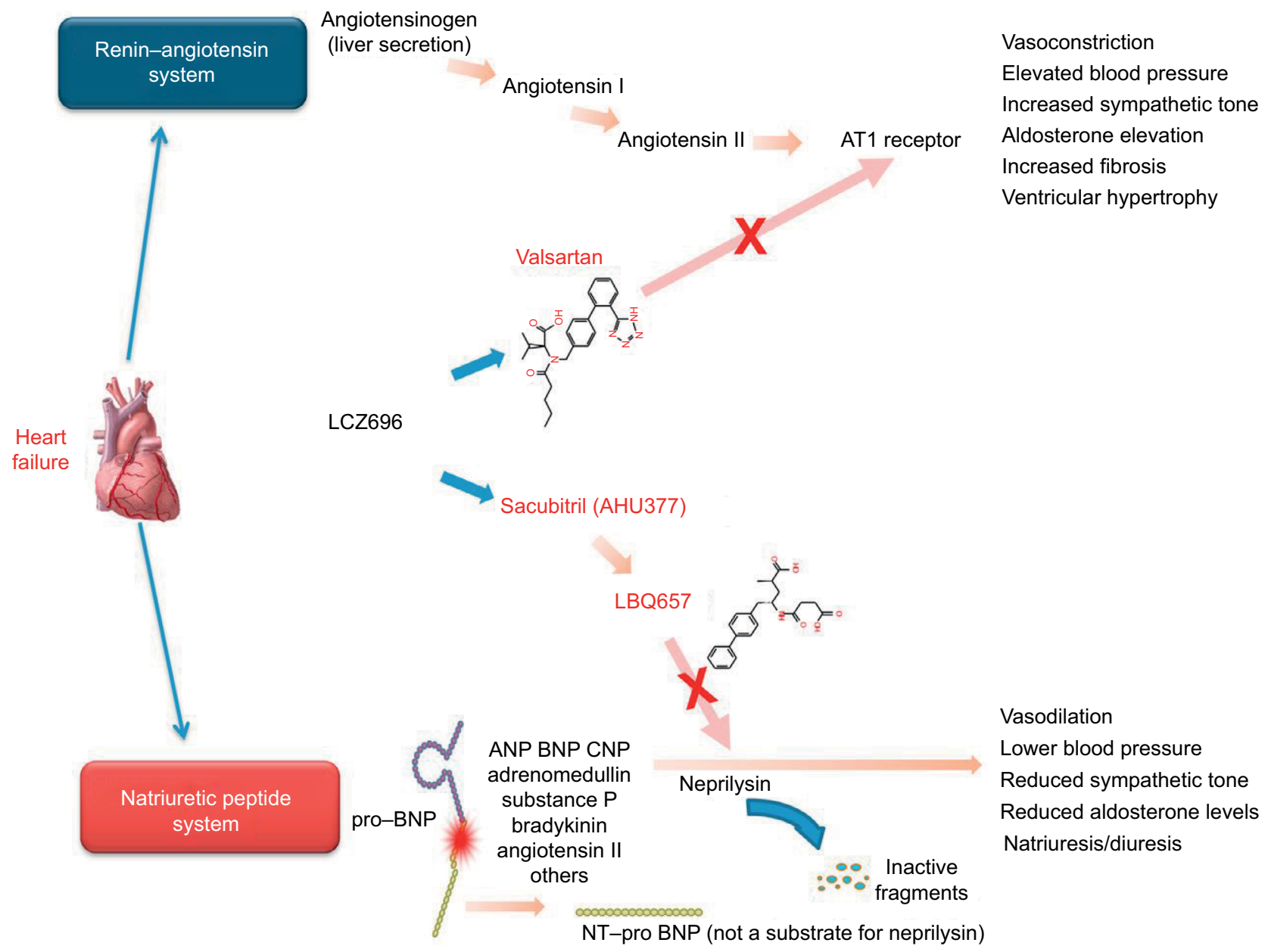

Figure I Mechanism of action of sacubitril/valsartan in heart failure.

Notes: Heart failure stimulates both the renin-angiotensin system and the natriuretic peptide system. LCZ696 is composed of two molecular moieties, the angiotensin receptor blocker valsartan and the neprilysin inhibitor prodrug sacubitril (AHU377). Valsartan blocks the ATI receptor. Sacubitril is converted enzymatically to the active neprilysin inhibitor LBQ657, which inhibits neprilysin, an enzyme that breaks down ANP, BNP, and CNP, as well as other vasoactive substances. NT-proBNP is not a substrate for neprilysin. Reprinted from JACC: Heart Failure, Volume 2/Edition 6, Vardeny O, Miller R, Solomon SD, Combined neprilysin and renin-angiotensin system inhibition for the treatment of heart failure, Pages 663-670, Copyright (2014), with permission from Elsevier. ${ }^{15}$

Abbreviations: ANP, atrial natriuretic peptide; ATI, angiotensin type I; BNP, brain (or B-type) natriuretic peptide; CNP, C-type natriuretic peptide; NT-proBNP, N-terminal pro-BNP.

from the PARADIGM-HF trial and subsequent secondary analyses incited a lot of excitement in the HF community, as the discovery of sacubitril/valsartan proves momentous in the relatively stagnant field of HF pharmacotherapy.

\section{International consensus recommendation for sacubitril/ valsartan in HF}

Following the trial, the USA FDA reviewed LCZ696 under its priority review program with a fast-track designation and approved this drug for the treatment of all NYHA II-IV HFrEF patients in July 2015. ${ }^{19}$ The USA FDA labeling is overarching, recommending the medication for all NYHA function class II-IV HFrEF patients with serum potassium $\leq 5.2 \mathrm{mmol} / \mathrm{L}$, and no contraindication or intolerance to ACEI/
ARB. The same year, the European Medical Agency approved the use of sacubitril/valsartan in adult patients for treatment of symptomatic chronic HFrEF. ${ }^{20}$

In their 2016 focused update on HF guidelines, the American College of Cardiology, the American Heart Association, and the Heart Failure Society of America recommended replacing an ACEI or ARB with an ARNI in patients with chronic symptomatic HFrEF, NYHA class II or III, currently tolerating an ACEI or ARB, to further reduce morbidity and mortality (class I recommendation). ${ }^{11}$ The guideline also recommended against concomitant use of ARNI with ACEI or within 36 hours of last ACEI dose, as it can precipitate angioedema (class III recommendation). ${ }^{11}$

The Canadian Cardiovascular Society was the first to incorporate the use of sacubitril/valsartan in HF patients. ${ }^{12}$ In 
Table I The PARADIGM-HF trial ${ }^{9,16}$

\begin{tabular}{|c|c|c|c|}
\hline Study (first author, year); Aim & Study population & Design & Outcomes \\
\hline $\begin{array}{l}\text { PARADIGM-HF (McMurray et al, } \\
\text { 20I4); }{ }^{9} \text { comparison of efficacy of } \\
\text { LCZ696 versus enalapril in patients } \\
\text { with HFrEF (LVEF } \leq 35 \% \text { ) }\end{array}$ & $\begin{array}{l}\mathrm{n}=8,442 ; \text { mean age } 64 \\
\text { years; mean LVEF } 29 \pm 6 \% \text {; } \\
70 \% \text { NYHA class II; } 78 \% \\
\text { male; } 66 \% \text { white; } 5 \% \text { black; } \\
60 \% \text { ICM; I00\% on either } \\
\text { an ACEI or an ARB; } 93 \% \\
\text { on beta-blockers }\end{array}$ & $\begin{array}{l}\text { Multicenter, randomized, } \\
\text { double-blind study of LCZ696 } \\
200 \mathrm{mg} \text { twice daily versus } \\
\text { enalapril } 10 \mathrm{mg} \text { twice daily for } \\
\text { a median of } 27 \text { months } \\
\text { 2-week enalapril run-in } \\
\text { period and a 4-6-week } \\
\text { LCZ696 run-in period prior } \\
\text { to randomization to ensure } \\
\text { target dose tolerability }\end{array}$ & 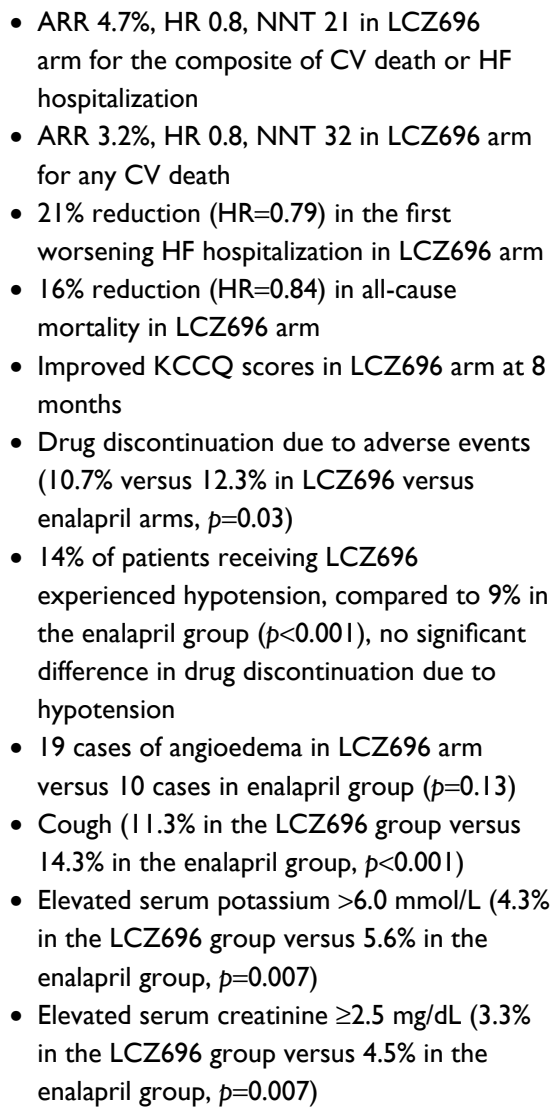 \\
\hline
\end{tabular}

Notes: Yandrapalli S, Aronow WS, Mondal P, et al. Limitations of sacubitril/valsartan in the management of heart failure. American Journal of Therapeutics. Volume 24, Issue 2: Pages 234-239. ${ }^{16}$

Abbreviations: ACEI, angiotensin-converting enzyme inhibitor; ARB, angiotensin receptor blocker; ARR, absolute risk reduction; CV, cardiovascular; HF, heart failure; HFrEF, heart failure with reduced ejection fraction; HR, hazard ratio; ICM, ischemic cardiomyopathy; KCCQ, Kansas City Cardiomyopathy Questionnaire; LVEF, left ventricular ejection fraction; NNT, number needed to treat; NYHA, New York Heart Association; PARADIGM-HF, Prospective Comparison of Angiotensin ReceptorNeprilysin Inhibitor with Angiotensin-Converting Enzyme Inhibitor to Determine Impact on Global Mortality and Morbidity in Heart failure.

their 2014 update on Heart Failure Management Guidelines, the Canadian guidelines recommend the use of LCZ696 in place of an ACEI or an ARB in patients with mild to moderate $\mathrm{HF}, \mathrm{LVEF}<40 \%$, an elevated natriuretic peptide level or hospitalization for HF in the past 12 months, a serum potassium $<5.2 \mathrm{mmol} / \mathrm{L}$, an estimated glomerular filtration rate (eGFR) $\geq 30 \mathrm{~mL} / \mathrm{min}$, and treated with appropriate doses of guideline-directed medical therapy, with close surveillance of serum potassium and creatinine (Conditional Recommendation; High-Quality Evidence). ${ }^{12}$ The Canadian HF guidelines limit the use of ARNI in patients who met the PARADIGM trial entry criteria.

The 2016 European Society of Cardiology (ESC) HF guidelines recommend the use of sacubitril/valsartan as an ACEI replacement to further reduce the risk of death and HF hospitalization in ambulatory patients with HFrEF (LVEF <35\%) who remain symptomatic despite optimal treatment with ACEI, a beta-blocker, and an MRA (class IB recommendation). ${ }^{13}$ In contrast to the American College of Cardiology, the American Heart Association, and the Heart Failure Society of America HF guidelines, the ESC guidelines specify having an LVEF cutoff of $35 \%$ prior to initiation of sacubitril/valsartan. The ESC guidelines also require the patient to be tried on an MRA before initiating an ARNI.

\section{Concerns with PARADIGM-HF and answers}

Current recommendations for the use of sacubitril/valsartan in HFrEF patients are based on the results of one trial, the PARADIGM-HF. Although this study is the largest and a globally represented high-quality clinical trial in HFrEF patients that evaluated key outcomes, it is not exempt from criticism. Since the publication of the original trial results in 2014, several physicians and researchers have raised 
apprehensions regarding the generalizability of the trial results with regards to the study design, the effect of target medication doses achieved on clinical outcomes, study population representativeness of the real-world HFrEF patients, and the effect of ethnic and geographic variations on outcomes. ${ }^{16}$

PARADIGM-HF was initiated without a Phase II safety trial, and hence, the investigators employed steps to test the tolerability of LCZ696 before randomization with a run-in phase for enalapril and then for LCZ696. ${ }^{10}$ More than $10 \%$ of the original study population dropped out during the LCZ696 run-in phase. ${ }^{9}$ Concern existed regarding randomizing only the patients who tolerated LCZ696 during the run-in phase of the trial, as such a design can exclude patients who are intolerant to the drug and can affect study outcomes and external validity. However, a post hoc analysis of the trial data showed that run-in noncompletion did not affect the magnitude of the treatment benefit of LCZ696 over enalapril. ${ }^{21,22}$ Low blood pressure, low eGFR, and more advanced HF (higher NTproBNP level) were associated with higher risk for run-in noncompletion, suggesting that these patients should undergo closer monitoring during the uptitration of LCZ696 or the conversion of patients from enalapril to LCZ696. ${ }^{21,22}$

The mean target dose achieved for enalapril (18.9 mg/ day) was lower than the maximum recommended dose in HF, whereas the maximum recommended target dose was achieved for sacubitril/valsartan. ${ }^{16}$ This raises the concern as to whether the maximal dose of valsartan produced more RAAS blockade leading to better clinical outcomes when compared to a moderate dose of enalapril, as no trials showed head-to-head comparison of the study doses of valsartan and enalapril as used in the PARADIGM-HF. ${ }^{16}$ The mean target dose for enalapril achieved in most placebo-controlled landmark HF trials providing significant $\mathrm{CV}$ benefit was also $<20 \mathrm{mg}$ /day $(16.6 \mathrm{mg} /$ day in the Studies of Left Ventricular Dysfunction and $18.4 \mathrm{mg} /$ day in the Cooperative North Scandinavian Enalapril Survival Study). ${ }^{21}$ It is important to note that in routine clinical practice, $<30 \% \mathrm{HF}$ patients achieved the target dose of enalapril due to complications including hypotension and hyperkalemia. ${ }^{21}$ Although LCZ696-treated patients had greater risk of developing symptomatic hypotension ( $14 \%$ versus $9 \%, p<0.001$; Table 1$)$, it did not result in significantly more medication discontinuation than enalapril patients, suggesting better tolerance with LCZ696. ${ }^{9,21}$

Black patients have been less represented in HF trials, although they have poorer outcomes. The lower proportion of black patients in the PARADIGM-HF trial (5\%) may have limited the ability of this trial to detect a significant difference in the frequency of angioedema with LCZ696. ${ }^{16}$ This is particularly concerning, as black patients had significantly more instances of angioedema compared to enalapril in the Omapatrilat Cardiovascular Treatment versus Enalapril trial. ${ }^{16}$ Future studies should shed light on this association.

\section{Profile of sacubitril/valsartan in HF and patient selection perspectives}

In this section, we will discuss the profile of sacubitril/ valsartan in HF with regards to patient characteristics and future perspectives for its use, with a mention of currently undergoing clinical trials. Registry data on HFrEF patients from Acute Decompensated Heart Failure National Registry (ADHERE) and Organized Program to Initiate Lifesaving Treatment in Hospitalized Patients With Heart Failure (OPTIMIZE-HF) were used to provide comparisons with PARADIGM-HF patient population in available baseline demographic characters (Table 2). ${ }^{9,23,24}$

OPTIMIZE-HF is a large US national registry and performance improvement program for patients hospitalized for $\mathrm{HF}^{23} \mathrm{ADHERE}$ is a large multicenter registry designed to compile a large clinical database on the clinical characteristics, management, and outcomes of patients hospitalized for $\mathrm{HF}$ across the USA. ${ }^{24}$ When compared to the HFrEF population in these registries, the PARADIGM-HF study population is relatively younger and male dominant. Additionally, the population has less black subjects, higher mean LVEF, lower SBP at randomization, excluded chronic kidney disease (CKD) stage 4 and 5 (eGFR $<30 \mathrm{~mL} / \mathrm{min} / 1.73 \mathrm{~m}^{2}$ ) patients, and most patients in NYHA functional class II, with almost no NYHA class IV patients. ADHERE and OPTIMIZE-HF represent real-world US HF patients; hence, these differences in patient characteristics have the potential to affect clinical outcomes in real-world HFrEF patients.

\section{Age}

Similar to the real-world HF population, mortality and HF hospitalization rate increased with age in PARADIGM-HF. ${ }^{9}$ The median patient age in the trial was 63.8 years, which is lower than the real-world HFrEF population and those in ADHERE (mean 70 years) and OPTIMIZE-HF (mean 70 years) registry (Table 2).9,23,24 This difference could have important clinical considerations with regards to better outcomes noticed in this relatively younger ambulatory HFrEF cohort. It is interesting to note that the clinical benefit observed in the sacubitril/valsartan group was consistent across prespecified age groups including patients $<55$ and $>75$ years, when compared to enalapril. ${ }^{25} \mathrm{HF}$ is a progressive 
Table 2 Baseline patient characteristics in various reduced ejection fraction heart failure patient study groups

\begin{tabular}{|c|c|c|c|}
\hline Study population characteristics & OPTIMIZE- HF ${ }^{23}$ & ADHERE $^{24}$ & PARADIGM-HF9 \\
\hline Patients with HFrEF (among those & 20,118 & 25,865 & 8,442 \\
\hline \multicolumn{4}{|l|}{ with data on LVEF assessment) } \\
\hline Mean age (SD), years & $70(14)$ & $70(14)$ & $63.8(11.5)$ \\
\hline Sex & $62 \%$ male & $60 \%$ male & $78 \%$ male \\
\hline \multirow[t]{3}{*}{ Race } & & $72 \%$ Caucasian & $66 \%$ Caucasian \\
\hline & $21 \%$ black & $22 \%$ black & $5 \%$ black \\
\hline & & & $18 \%$ Asian \\
\hline \multirow[t]{2}{*}{ Etiology of HF } & $54 \%$ ICM & $59 \%$ population & $60 \%$ ICM \\
\hline & I7\% hypertensive HF & had CAD & I I.5\% Hypertensive HF \\
\hline Hypertension & $66 \%$ & $69 \%$ & $70 \%$ \\
\hline Diabetes mellitus & $39 \%$ & $40 \%$ & $34.6 \%$ \\
\hline CKD & & $26 \%$ & - \\
\hline Atrial fibrillation & $28 \%$ & & $37 \%$ \\
\hline LVEF $\pm S D(\%)$ & $24 \pm 8$ & & $29.5 \pm 6.2$ \\
\hline Mean SBP $(\mathrm{mmHg})$ & $|35 \pm 3|$ & & $121(15)$ \\
\hline \multirow[t]{4}{*}{ NYHA functional class } & $44 \%$ class IV & & $5 \%$ class 1 \\
\hline & & & $70 \%$ class II \\
\hline & & & $24 \%$ class III \\
\hline & & & $0.7 \%$ class IV \\
\hline
\end{tabular}

Abbreviations: ADHERE, Acute Decompensated Heart Failure National Registry; CAD, coronary artery disease; CKD, chronic kidney disease; HF, heart failure; HFrEF, heart failure with reduced ejection fraction; ICM, ischemic cardiomyopathy; LVEF, left ventricular ejection fraction; NYHA, New York Heart Association; OPTIMIZEHF, Organized Program to Initiate Lifesaving Treatment in Hospitalized Patients With Heart Failure; PARADIGM-HF, Prospective Comparison of Angiotensin ReceptorNeprilysin Inhibitor with Angiotensin-Converting Enzyme Inhibitor to Determine Impact on Global Mortality and Morbidity in Heart failure; SBP, systolic blood pressure.

disease and advances in severity with age, so initiation of sacubitril/valsartan at a younger age in HFrEF patients who have a less-advanced disease stage will provide more clinical and survival benefit.

\section{Sex}

The study population in PARADIGM-HF is predominantly male $(78 \%)$ with a rate higher than that observed for HFrEF patients in the ADHERE (60\%) and OPTIMIZE-HF registries (62\%) (Table 2). ${ }^{9}$ This proportion is higher when compared to real-world HF patients in whom HFrEF is almost equally prevalent sex-wise. However, in prespecified subgroup analysis of the PARADIGM-HF, the clinical benefit of sacubitril/ valsartan was not affected by sex. ${ }^{9}$ ARNI can be used without reservation in HFrEF patients of either sex.

\section{Race/ethnicity}

As mentioned earlier, caution should be exercised when prescribing ARNI to black HFrEF patients, as available safety data about angioedema is limited in this underrepresented population in the PARADIGM-HF., ${ }^{9,16}$ The prospective comparison of ARNI with ACE inhibitor to determine the novel beneficial treatment value in Japanese HF patients (PARALLEL-HF) trial is now being conducted to assess the safety and efficacy of sacubitril-valsartan in Japanese HFrEF population. This is a multicenter, randomized, double-blind study with similar design, phases, inclusion and exclusion criteria as in PARADIGM-HF trial (Table 3) ${ }^{26}$

\section{Regional demographic variations}

Significant regional differences in baseline demographics such as age, symptoms, comorbidities, background therapies, and event rates between patients enrolled from different geographic locations exist in the PARADIGM-HF cohort. ${ }^{27}$ A post hoc analysis of the original trial demonstrated a consistent clinical benefit of sacubitril/valsartan across geographic regions, despite having significant baseline regional demographic variations. ${ }^{27}$ These findings are encouraging for the global use of this novel pharmacotherapy in HFrEF patients to provide reduction in HF morbidity and mortality across the world.

\section{NYHA functional class}

Majority of the study population in the PARADIGM-HF consisted of NYHA class II patients (70\%). ${ }^{9}$ A nominally significant interaction between NYHA class at randomization and the effect of treatment was seen for the primary endpoint (better outcomes with NYHA class I-II; $p=0.03$; without adjustment for multiple comparisons), but not for the interaction between NYHA class and the effect on death from $\mathrm{CV}$ causes $(p=0.76){ }^{9}$ Although the interaction is nominal, it confers a greater $\mathrm{CV}$ benefit to sacubitril/valsartan when initiated early in the course of HFrEF. With almost $75 \%$ patients in the trial classified as either NYHA class I or II, the power of this group to identify an interaction if present may be increased. Nevertheless, initiation of sacubitril/valsartan early in the disease course of HFrEF is encouraged. 


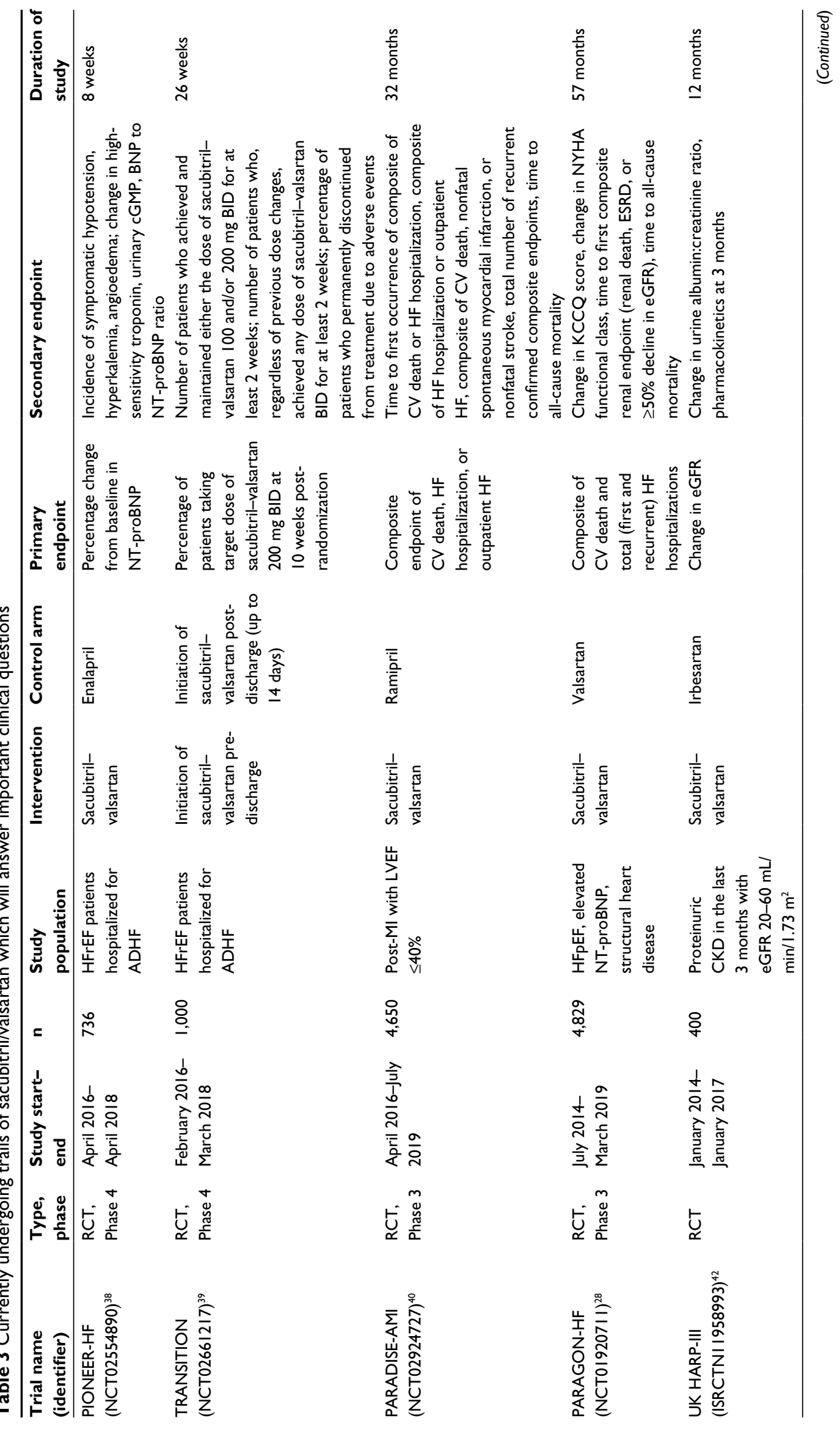




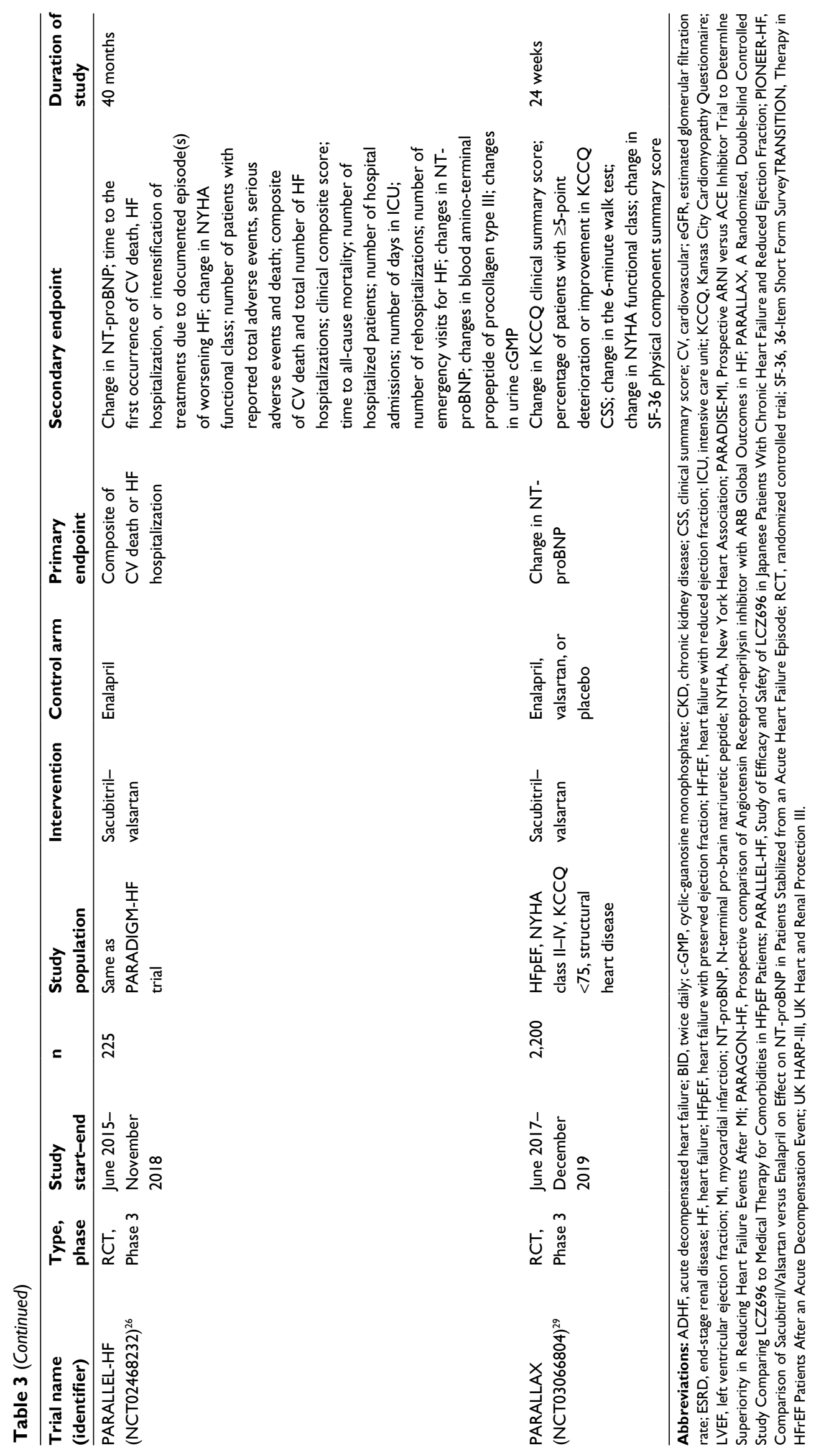




\section{Type of HF}

Sacubitril/valsartan is currently approved for use in chronic HFrEF patients. Having demonstrated safety in HFpEF population and efficacy in hypertensive patients in earlier trials, sacubitril/valsartan has the potential to show substantial clinical benefit in the HFpEF population, since hypertension is an important risk factor for HFpEF. The Phase III, randomized controlled, Prospective comparison of Angiotensin Receptor-neprilysin inhibitor with ARB Global Outcomes in HF with preserved ejection fraction (PARAGON-HF) trial is evaluating the effects of sacubitril/valsartan versus valsartan on the primary composite outcome of $\mathrm{CV}$ death and HF hospitalization in HFpEF patients and is expected to be completed in 2019 (Table 3). ${ }^{28}$ The PARAGON-HF will also compare treatment benefit of sacubitril/valsartan versus valsartan on functional class, change in Kansas City Cardiomyopathy Questionnaire score, and time to deterioration in renal function. ${ }^{28}$ The randomized, double-blind controlled study comparing LCZ696 to medical therapy for comorbidities in HFpEF patients (PARALLAX) is a 24-week, multicenter, parallel-group, active controlled study which will evaluate the effect of sacubitril/valsartan on NT-proBNP levels, symptoms, exercise function, and safety, compared to individualized medical management of comorbidities (with enalapril, valsartan, or placebo) in HFpEF patients (Table 3). ${ }^{29}$

\section{Etiology of HF}

HFrEF has different etiologies depending on age, sex, geography, and race. Clinical outcomes and response to medical therapies in HFrEF patients will vary depending on etiology. In the PARADIGM-HF trial, $60 \%$ of the study population had ischemic cardiomyopathy (ICM), which is comparable to that observed in HFrEF patients in the ADHERE and the OPTIMIZE-HF registries (Table 2). ${ }^{9,23,24}$ This raises concern of the benefit of sacubitril/valsartan in patients with non-ICM (NICM). A post hoc analysis of the PARADIGM-HF classified the study population based on etiology of HF into patients with ICM (60\%) and NICM (40\%). ${ }^{30}$ NICM consisted of idiopathic HF (19\%), hypertensive HF (11.5\%), and other causes of HF $(9.5 \%))^{30}$ The investigators noted that patients with NICM were younger, more likely female, and had higher NT-proBNP levels than those with ICM. ${ }^{30}$ With regards to primary composite endpoint and $\mathrm{CV}$ death, the benefit of sacubitril/valsartan over enalapril was consistent across the different etiology subgroups. ${ }^{30}$ The authors concluded that sacubitril/valsartan is effective in both ICM and NICM including idiopathic and hypertensive causes of NICM. These findings have important considerations when using this ARNI in real-world HFrEF patients and suggest that the complex pathophysiologic interactions of ARNI with the RAAS are independent of the type of insult resulting in HF.

\section{Left ventricular ejection fraction}

In the PARADIGM-HF trial, the risk of all clinical outcomes increased with decreasing LVEF (mean 29.5\%), and LVEF was noted to be a strong predictor of outcomes. ${ }^{9}$ In a post hoc analysis, sacubitril/valsartan was effective across the LVEF spectrum and there was no evidence of heterogeneity for the primary endpoint, CV death, HF hospitalization, or all-cause mortality. ${ }^{31}$ These findings suggest that the benefit of sacubitril/valsartan is not LVEF dependent and may have implications in its testing in $\mathrm{HFpEF}$ patients.

\section{Intracardiac devices}

Patients with cardiac devices were underrepresented in the PARADIGM-HF trial. Around 15\% had implantable cardioverter-defibrillator and around 7\% patients had cardiac resynchronization therapy. ${ }^{9}$ Despite this limitation, the magnitude of clinical benefit of sacubitril/valsartan on sudden death did not differ in patients with or without an implantable cardioverter-defibrillator. ${ }^{32} \mathrm{ARNI}$ use will provide additional $\mathrm{CV}$ and survival benefit in symptomatic HFrEF patients, irrespective of them having an intracardiac device.

\section{ADHF and post-acute myocardial infarction patients}

PARADIGM-HF excluded patients who had an acute coronary event within the last 3 months of randomization. ${ }^{9}$ The benefit of initiating or uptitrating neurohormonal antagonist during an episode of ADHF is not clear. ${ }^{33}$ However, it is well known that initiation of beta-blocker, ACEI, and an MRA after acute myocardial infarction (MI) with associated left ventricle systolic dysfunction reduces the rate of hospitalization for HF and mortality. ${ }^{34-36}$ In a post hoc analysis of the PARADIGMHF trial, the benefit of sacubitril-valsartan in reducing CV mortality or hospitalization for HF was consistent in all patient subgroups, with no significant difference in patients with no prior HF hospitalization compared to those with recent HF hospitalization ( $<3$ months from randomization). ${ }^{37}$

Although available data do not support the use of this ARNI in ADHF patients and in post-acute MI patients, ongoing trials will shed more light. Comparison of Sacubitril/ Valsartan versus Enalapril on Effect on NT-proBNP in Patients Stabilized from an Acute Heart Failure Episode (PIONEERHF) is a multicenter, randomized, double-blind, 8-week study, 
with the aim being to evaluate the effect of sacubitril-valsartan versus enalapril on changes in NT-proBNP in HFrEF patients who have been stabilized following hospitalization for ADHF. The primary outcome is the percentage change in NT-proBNP from baseline to weeks 4 and 8 (Table 3 ). ${ }^{38}$

The Comparison of Pre- and Post-discharge Initiation of LCZ696 Therapy in HFrEF Patients After an Acute Decompensation Event (TRANSITION) trial is a multicenter, randomized, open-label study, with an estimated enrollment of 1,000 patients, aiming to compare the initiation of sacubitril/ valsartan after stabilization of patients with ADHF at any point prior to discharge with post-discharge initiation (up to 14 days). The primary outcome is the percentage of patients who are receiving sacubitril/valsartan $200 \mathrm{mg}$ twice daily at 10 weeks after randomization (Table 3). ${ }^{39}$ This trial will also evaluate the safety of initiation of sacubitril/valsartan in $\mathrm{ADHF}$ and the percentage of patients who permanently discontinued from treatment due to adverse events. ${ }^{39}$

The Prospective ARNI versus ACE Inhibitor Trial to DetermIne Superiority in Reducing Heart Failure Events After MI (PARADISE-MI) trial is a Phase III, randomized controlled study conducted in post-acute MI patients without prior chronic HF, with $\mathrm{LVEF}<40 \%$, and/or pulmonary congestion at the time of randomization. The PARADISE-MI trial will evaluate the benefit of sacubitril/valsartan versus ramipril in reducing the occurrence of the primary composite endpoint of $\mathrm{CV}$ death, HF hospitalization, and outpatient HF (time-to-first event analysis), as shown in Table $3 .{ }^{40}$

\section{CKD and hyperkalemia}

HFrEF patients frequently have CKD with varying degrees of eGFRs. Worsening renal function and hyperkalemia can limit the successful use or uptitration of RAAS blockade agents in HF patients. ${ }^{41}$ The PARADIGM-HF trial excluded patients with eGFR $<30 \mathrm{~mL} / \mathrm{min} / 1.73 \mathrm{~m}^{2}$, thereby limiting safety data on its use in CKD stage 4 and 5 patients. Interestingly, it was observed that LCZ696-treated patients had significantly less instances of elevated serum creatinine $\geq 2.5 \mathrm{mg} / \mathrm{dL}(3.3 \%$ in the LCZ696 group versus $4.5 \%$ in the enalapril group, $p=0.007$ ) compared to enalapril-treated patients. The UK Heart and Renal Protection III (UK HARP-III) trial will compare LCZ696 against irbesartan (an ARB) in patients with proteinuric CKD (eGFR $20-60 \mathrm{~mL} / \mathrm{min} / 1.73 \mathrm{~m}^{2}$ ) to assess the short-term safety and efficacy of LCZ696 in this population with respect to changes in eGFR (Table 3). ${ }^{42}$ If the results from the UK HARP-III trial favor LCZ696, longer duration studies to evaluate the effects of LCZ696 in delaying the progression of CKD to end-stage renal disease should be planned.
With regards to severe hyperkalemia, LCZ696-treated patients had lower instances of elevated serum potassium $>6 \mathrm{mmol} / \mathrm{L}$ (4.3\% in the LCZ696 group versus $5.6 \%$ in the enalapril group, $p=0.007$; Table 1). ${ }^{9}$ Moreover, among patients treated with an MRA, severe hyperkalemia was more likely with enalapril treatment than with LCZ696 (3.1 versus 2.2 per 100 patient-years, $p=0.02$ ), suggesting that LCZ696 might decrease the risk of hyperkalemia compared to enalapril, when added to MRA therapy in patients with HFrEF. ${ }^{43}$ Although further safety data will be available from currently ongoing trials, sacubitril/valsartan therapy might facilitate the uptitration of other RAAS blocking agents with proven mortality benefit in HFrEF patients without increasing the incidence of dose-limiting adverse events.

\section{Diabetes mellitus}

In the PARADIGM-HF trial, it was observed that patients with diabetes and pre-diabetes had a higher risk of the primary composite outcome. ${ }^{9}$ Sacubitril/valsartan reduced the risk of $\mathrm{CV}$ death and $\mathrm{HF}$ hospitalizations compared with enalapril, irrespective of the glycemic status. ${ }^{44} \mathrm{~A}$ recently published post hoc analysis of the PARADIGM-HF trial found that sacubitril/valsartan decreased hemoglobin A1C levels by $0.26 \%$ during the first year of follow-up, compared to a $0.16 \%$ reduction with enalapril $(p=0.0023) .{ }^{45}$ Over 3 years, HbAlc levels remained persistently lower in patients treated with sacubitril/valsartan compared to enalapril, with an overall reduction of $0.14 \%$ (95\% CI: $0.06-0.23$, $p=0.0055) .{ }^{45}$ In addition, $29 \%$ fewer sacubitril/valsartantreated patients initiated insulin therapy to achieve glycemic control ( $7 \%$ versus $10 \%$ patients, hazard ratio $0.71,95 \% \mathrm{CI}$ : $0.56-0.90 ; p=0.0052) .{ }^{45}$ These results are interesting as they hypothesize an additional metabolic benefit and emphasize the extracardiac pleiotropic effects of sacubitril/valsartan in HFrEF patients in addition to its compelling CV efficacy.

\section{Eligibility of the real-world HF patients for sacubitril/valsartan use}

The US FDA labeling for sacubitril/valsartan is very broad, recommending this medication for all NYHA function class II-IV HFrEF patients with serum potassium $\leq 5.2 \mathrm{mmol} / \mathrm{L}$ and no contraindication or intolerance to ACEI/ARB. ${ }^{19}$ Differences exist between international consensus guidelines for ARNI indications in HFrEF, which might affect real-world patient groups who will be prescribed this drug. Also, concerns exist regarding the generalizability of the results of PARADIGM-HF to the entire real-world HFrEF patients, as the PARADIGM-HF study population represents 
only a minority of the real-world HFrEF population. Several researchers investigated the differences in eligibility of sacubitril/valsartan initiation in the real-world HFrEF population based on FDA labeling versus PARADIGM-HF enrollment criteria.

Parikh et al evaluated patient population from the Get With The Guidelines-Heart Failure (GWTG-HF) registry to characterize patients' eligibility and potential barriers for sacubitril/valsartan initiation according to the criteria set forth in FDA labeling and PARADIGM-HF. ${ }^{46}$ The GWTGHF registry is composed of patients who were admitted for worsening HF or developed significant HF symptoms during a hospitalization. ${ }^{46}$ Among 28,932 hospitalizations of HFrEF patients, 20,083 (69\%) patients were eligible for sacubitril/ valsartan initiation based on FDA labeling and 11,018 (38\%) based on PARADIGM-HF criteria (including natriuretic peptide cutoffs), as shown in Table $4 .{ }^{46}$ Of the 20,083 who were eligible based on FDA criteria, only $55 \%$ would have been eligible for sacubitril/valsartan initiation based on
PARADIGM-HF criteria. Based on ESC guidelines, this number would go down further, as only $41 \%$ patients in the PARADIGM-HF eligible group were on an MRA. The most common reasons for ineligibility (using PARADIGM-HF criteria) were: $\mathrm{LVEF}>35 \%$, discharge SBP $<100 \mathrm{mmHg}$, and not being on ACEI/ARB therapy. ${ }^{21}$ Patients eligible for sacubitril/valsartan based on PARADIGM-HF criteria had significantly lower 30-day and 1-year mortality rates, compared to those who were excluded based on FDA-labeling or PARADIGM-HF entry criteria. ${ }^{46}$

Perez et al performed a single-center chart review of 210 NYHA class II-IV patients admitted to their HF service and had post-discharge follow-up, to determine the eligibility of sacubitril/valsartan initiation using FDA approval criteria and PARADIGM-HF trial enrollment criteria. ${ }^{47}$ Of the 149 (71\% total) patients who were eligible based on FDA labeling, only 54 (26\% total) were eligible based on PARADIGMHF enrollment criteria (Table 4). ${ }^{47}$ The authors found that the patients who did not meet PARADIGM-HF enrollment

Table 4 Studies evaluating the real-world eligibility of sacubitril/valsartan in HFrEF patients

\begin{tabular}{|c|c|c|c|c|}
\hline Population & $\begin{array}{l}\text { HFrEF patients who } \\
\text { were admitted for } \\
\text { worsening HF or } \\
\text { developed significant } \\
\text { HF symptoms during } \\
\text { a hospitalization (from } \\
\text { The Get With The } \\
\text { Guidelines-Heart Failure } \\
\text { Registry) }\end{array}$ & $\begin{array}{l}\text { NYHA class II-IV HF } \\
\text { patients who were } \\
\text { discharged from the HF } \\
\text { services of the Cleveland } \\
\text { Clinic and had post- } \\
\text { discharge follow-up }\end{array}$ & $\begin{array}{l}\text { Ambulatory NYHA } \\
\text { class II-IV chronic } \\
\text { HFrEF patients in the } \\
\text { Swedish Heart Failure } \\
\text { Registry (SwedeHF) }\end{array}$ & $\begin{array}{l}\text { Chronic HFrEF patients } \\
\text { with available NT- } \\
\text { proBNP measurements } \\
\text { and on target doses } \\
\text { ACEI/ARB referred to a } \\
\text { community HF clinic in } \\
\text { the UK }\end{array}$ \\
\hline Total no. of patients & 28,932 & 210 & 12,914 & 1,396 \\
\hline $\begin{array}{l}\text { Number of patients } \\
\text { meeting FDA labeling } \\
\text { (\%) for sacubitril/ } \\
\text { valsartan initiation }\end{array}$ & $20,083(69 \%)$ & 149 (7|\%) & $6,452(50 \%)$ & - \\
\hline $\begin{array}{l}\text { Number of patients } \\
\text { meeting PARADIGM- } \\
\text { HF criteria (\%) }\end{array}$ & II,0|8 (38\%) & $54(26 \%)$ & $5,015(39 \%)$ & 172 (2I\%) \\
\hline $\begin{array}{l}\text { Ratio of PARADIGM- } \\
\text { HF like patients to } \\
\text { FDA labeling eligible } \\
\text { patients }\end{array}$ & $55 \%$ & $37 \%$ & $78 \%$ & - \\
\hline $\begin{array}{l}\text { Reason for exclusion } \\
\text { based on FDA labeling }\end{array}$ & $\begin{array}{l}\text { Existing contraindication to } \\
\text { ACEI/ARB; low discharge } \\
\text { SBP }\end{array}$ & $\mathrm{N} / \mathrm{A}$ & $\begin{array}{l}\text { Not on at least enalapril } \\
10 \mathrm{mg} \text { daily or equivalent }\end{array}$ & - \\
\hline $\begin{array}{l}\text { Reason for exclusion } \\
\text { from PARADIGM-HF }\end{array}$ & $\begin{array}{l}\text { LVEF } 35 \%-40 \% \text {, low } \\
\text { discharge SBP }<100 \mathrm{mmHg}\end{array}$ & $\begin{array}{l}\mathrm{SBP} \leq 100 \mathrm{mmHg}, \text { eGFR } \leq 30 \\
\mathrm{~mL} / \mathrm{min} / \mathrm{I} .73 \mathrm{~m}^{2} \text {, not on } \mathrm{ACEl} \\
\text { or } \mathrm{ARB} \text {, not on beta-blocker, } \\
\text { serum } \mathrm{K} \geq 5.2 \mathrm{mmol} / \mathrm{L}\end{array}$ & $\begin{array}{l}\text { Low natriuretic peptide } \\
\text { level, SBP }<100 \mathrm{mmHg} \text {, } \\
\text { eGFR } \leq 30 \mathrm{~mL} / \mathrm{min} / \mathrm{l} .73 \\
\mathrm{~m}^{2} \text {, serum } \mathrm{K}>5.2 \mathrm{mmol} / \mathrm{L}\end{array}$ & $\begin{array}{l}\text { Not on target dosing of } \\
\text { ACEI/ARB, lack of limiting } \\
\text { symptoms, plasma NT- } \\
\text { proBNP }<600 \mathrm{ng} / \mathrm{mL}\end{array}$ \\
\hline
\end{tabular}

Abbreviations: ACEI, angiotensin-converting enzyme inhibitor; ARB, angiotensin receptor blocker; eGFR, estimated glomerular filtration rate; FDA, US Food and Drug Administration; HF, heart failure; HFrEF, heart failure with reduced ejection fraction; LVEF, left ventricular ejection fraction; NT-proBNP, N-terminal pro-brain natriuretic peptide; NYHA, New York Heart Association; PARADIGM-HF, Prospective Comparison of Angiotensin Receptor-Neprilysin Inhibitor with Angiotensin-Converting Enzyme Inhibitor to Determine Impact on Global Mortality and Morbidity in Heart failure; SBP, systolic blood pressure. 
criteria were sicker with higher NYHA functional class, lower SBP, greater NT-proBNP level, lower eGFR, and they were less likely to be on ACEI, beta-blocker, and an MRA. ${ }^{47}$ These findings reiterate the significant evidence gap with regards to FDA labeling of sacubitril/valsartan and proves concerning for the safety of sacubitril/valsartan as it can be used now in real-world HF population which was not represented in the PARADIGM-HF trial.

The above-mentioned analyses are primarily in hospitalized patients, whereas PARADIGM-HF enrolled ambulatory patients. European investigators analyzed registry data to establish the eligibility of ambulatory patients with HFrEF for treatment with sacubitril/valsartan. Simpson et al analyzed the Swedish Heart Failure Registry (SwedeHF) to determine the eligibility of patients with $\mathrm{HFrEF}$ for treatment with sacubitril/valsartan, according to the criteria used in PARADIGM-HF (Table 4). ${ }^{48}$ They identified 12,914 ambulatory patients with symptomatic (NYHA class II-IV) HFrEF (LVEF <40\%), of which 6,452 were treated with at least enalapril $10 \mathrm{mg}$ daily or equivalent. ${ }^{48}$ Of these 6,452 patients treated with enalapril $10 \mathrm{mg}$ daily or equivalent, $5,015(77.7 \%)$ were eligible for treatment with sacubitrilvalsartan using PARADIGM-HF inclusion criteria and the most common reason for ineligibility was low natriuretic peptide level (17.6\%). ${ }^{48}$ Very few ambulatory symptomatic HFrEF patients on enalapril were ineligible due to a low eGFR of $<30 \mathrm{~mL} / \mathrm{min} / 1.73 \mathrm{~m}^{2}(87 / 6,452 ; 1.3 \%)$ or serum potassium $>5.2 \mathrm{mmol} / \mathrm{L}(51 / 6,452 ; 0.7 \%) .{ }^{48}$ Based on these findings, $38.8 \%$ of the ambulatory SwedeHF patients with symptomatic HFrEF were eligible for sacubitril/valsartan initiation. The findings from this study make sense in a broader perspective, as ambulatory HF patients are less sick than hospitalized patients and have lower natriuretic peptide levels, higher eGFR, and lower serum potassium levels.

These findings are in contrast with those reported by Pellicori et al who studied patient population in a community HF referral clinic in the UK between 2001 and $2014 .{ }^{49}$ They identified 1,396 chronic HFrEF patients with LVEF $\leq 40 \%$ and available NT-proBNP measurements (Table 4). ${ }^{49}$ Of the 379 patients (27\% of total) who were on target dose of ACEI/ARB, only 172 patients (45\% of 379) were eligible for sacubitril-valsartan initiation based on PARADIGM-HF enrollment criteria. ${ }^{49}$ Lack of symptoms and low NT-proBNP $<600 \mathrm{ng} / \mathrm{L}$ levels were the most common reasons for ineligibility. ${ }^{49} \mathrm{~A}$ further 122 patients became eligible during followup. Based on this study, in a community-based chronic HFrEF population, only $21 \%(294 / 1,396)$ were eligible for sacubitril/ valsartan initiation based on PARADIGM-HF enrollment criteria, a number that increased to $60 \%(701 / 1,396)$ when background medications were ignored. ${ }^{49}$ However, it should be remembered that being on an $\mathrm{ACEI} / \mathrm{ARB}$ is a requirement prior to ARNI initiation. It is also important to note that this study is based on a single referral HF clinic, whereas the SwedeHF included a broader HFrEF population, which can explain the difference in the proportions of ambulatory patients who were eligible for sacubitril/valsartan initiation.

Zeymer et al compared PARADIGM-HF enrollment criteria with retrospective data from three German chronic HFrEF (LVEF <40\%) registries/studies and reported that patients enrolled in PARADIGM-HF trial represented about $50 \%$ of the general population of patients with HFrEF in these registries. ${ }^{50}$ It is interesting to note that only $5.3 \%-20.4 \%$ patients in the registries were treated with enalapril. Overall, it is evident that the patient population studied in the PARADIGM-HF does not represent the entire real-world HFrEF population largely because of the lack of symptoms (NYHA class I), not being on an ACEI/ARB, or low levels of natriuretic peptide. It is obvious from these findings that sacubitril/valsartan cannot be prescribed for the entire HFrEF population, given that a few major exclusions in the trial represent a significant number of real-world HFrEF population. It is also apparent that the differences in FDA labeling and consensus recommendations may result in this ARNI being used in a larger HFrEF population than it is evaluated in.

\section{Conclusion}

Although the PARADIGM-HF trial provided compelling evidence for the superiority of angiotensin receptor-NEP inhibition over enalapril in HFrEF patients, it is important to note that certain subgroups of patients who represent a significant proportion of the real-world HF population were underrepresented or excluded from the original trial. Real-world eligibility data suggest that only $20 \%-40 \%$ of the HFrEF patients will be eligible for sacubitril/valsartan initiation based on current guidelines. The differences in FDA labeling and international consensus recommendations may result in this ARNI being used in a larger HFrEF population than it is studied in or recommended for, raising important safety concerns. It is crucial to clarify this evidence gap to prevent unwarranted complications in HFrEF patients in whom sacubitril/valsartan will be prescribed. Variations in inter-consensus guidelines must be also clarified, as these differences might affect patient selection. Despite these reservations, sacubitril/valsartan is an important breakthrough for the management of HFrEF and should be used in appropriately selected patients to provide morbidity and mortality 
reduction. Available evidence suggests that sacubitril/valsartan might have a metabolic and nephroprotective benefit in HFrEF patients and proposes a significant $\mathrm{CV}$ benefit of this ARNI, irrespective of the type or etiology of HF. Ongoing trials testing this combination across various comorbidity profiles will expand the current indications of this gamechanger medication in the management of HF.

\section{Disclosure}

The authors report no conflicts of interest in this work.

\section{References}

1. Redfield MM. Heart Failure with preserved ejection fraction. $N$ Engl J Med. 2016;375(19):1868-1877.

2. Fonarow GC, Stough WG, Abraham WT, et al. Characteristics, treatments, and outcomes of patients with preserved systolic function hospitalized for heart failure: a report from the OPTIMIZE-HF Registry. J Am Coll Cardiol. 2007;50(8):768-77.

3. Braunwald $\mathrm{E}$. The war against heart failure: the Lancet lecture. Lancet. 2015;385(9970):812-824.

4. Mozaffarian D, Benjamin EJ, Go AS, et al. Heart disease and stroke statistics-2016 update: a report from the American Heart Association. Circulation. 2016;133(4):38-360.

5. Gheorghiade M, Pang PS. Acute heart failure syndromes. J Am Coll Cardiol. 2009;53(7):557-573.

6. Rudiger A, Harjola VP, Muller A, et al. Acute heart failure: clinical presentation, one-year mortality and prognostic factors. Eur J Heart Fail. 2005;7(4):662-670.

7. Fonarow GC, Abraham WT, Albert NM, et al. Association between performance measures and clinical outcomes for patients hospitalized with heart failure. JAMA. 2007;297(1):61-70.

8. RogerVL. Epidemiology of heart failure. Circ Res. 2013;113(6):646-659.

9. McMurray JJ, Packer M, Desai AS, et al. Angiotensin-neprilysin inhibition versus enalapril in heart failure. $N$ Engl $J$ Med. 2014;371(11):993-1004.

10. Yandrapalli S, Aronow WS, Mondal P, et al. The evolution of natriuretic peptide augmentation in management of heart failure and the role of sacubitril/valsartan. Arch Med Sci. 2017;13(5):1207-1216.

11. Yancy CW, Jessup M, Bozkurt B, et al. 2017 ACC/AHA/HFSA focused update of the $2013 \mathrm{ACCF} / \mathrm{AHA}$ guideline for the management of heart failure: a report of the American College of Cardiology/American Heart Association task force on clinical practice guidelines and the heart failure society of America. Circulation. 2017;136(6):e137-e161.

12. Moe GW, Ezekowitz JA, O'Meara E, et al. The 2014 Canadian Cardiovascular Society heart failure management guidelines focus update: anemia, biomarkers, and recent therapeutic trial implications. Can $J$ Cardiol. 2015;31(1):3-16.

13. Ponikowski P, Voors AA, Anker SD, et al. 2016 ESC Guidelines for the diagnosis and treatment of acute and chronic heart failure: the task force for the diagnosis and treatment of acute and chronic heart failure of the European Society of Cardiology (ESC). Eur J Heart Fail. 2016;18(8):891-975.

14. Gu J, Noe A, Chandra P, et al. Pharmacokinetics and pharmacodynamics of LCZ696, a novel dual-acting angiotensin receptor - neprilysin inhibitor (ARNi). J Clin Pharmacol. 2010;50(4):401-414.

15. Vardeny O, Miller R, Solomon SD. Combined neprilysin and reninangiotensin system inhibition for the treatment of heart failure. JACC Heart Fail. 2014;2(6):663-670.

16. Yandrapalli S, Aronow WS, Mondal P, et al. Limitations of sacubitril/valsartan in the management of heart failure. Am J Ther. 2017;24(2):234-239.
17. Packer M, McMurray JJ, Desai AS, et al. Angiotensin receptor neprilysin inhibition compared with enalapril on the risk of clinical progression in surviving patients with heart failure. Circulation. 2015;131(1):54-61.

18. McMurray J, Packer M, Desai AS, et al. A putative placebo analysis of the effects of LCZ696 on clinical outcomes in heart failure. Eur Heart J. 2015;36(7):434-439.

19. FDA news release: FDA approves new drug to treat heart failure. U.S. Food and Drug Administration; 2015. Available from: http://www.fda. gov/NewsEvents/Newsroom/PressAnnouncements/ucm453845.htm. Accessed June 12, 2017.

20. Summary of the Risk Management Plan (RMP) for Entresto (Sacubitril/ Valsartan). EMA; 2015. Available from: http://www.ema.europa.eu/ docs/en_GB/document_library/EPAR_-_Risk-management-plan_summary/human/004062/WC500194315.pdf. Accessed June 20, 2017.

21. Vinereanu D. Sacubitril-valsartan for heart failure: from devil's advocate to evidence-based medicine. Am J Ther. 2017;24(2):109-110.

22. Desai AS, Solomon S, Claggett B, et al. Factors associated with noncompletion during the run-in period before randomization and influence on the estimated benefit of LCZ696 in the PARADIGM-HF Trial. Circ Heart Fail. 2016;9(6):002735.

23. Fonarow GC, Stough WG, Abraham WT, et al. Characteristics, treatments, and outcomes of patients with preserved systolic function hospitalized for heart failure: a report from the OPTIMIZE-HF Registry. J Am Coll Cardiol. 2007;50(8):768-777.

24. Yancy CW, Lopatin M, Stevenson LW, et al. Clinical presentation, management, and in-hospital outcomes of patients admitted with acute decompensated heart failure with preserved systolic function: a report from the Acute Decompensated Heart Failure National Registry (ADHERE) Database. J Am Coll Cardiol. 2006;47(1):76-84.

25. Jhund PS, Fu M, Bayram E, et al. Efficacy and safety of LCZ696 (sacubitril-valsartan) according to age: insights from PARADIGM-HF. Eur Heart J. 2015;36:2576-2584.

26. Novartis. Study of efficacy and safety of LCZ696 in Japanese patients with chronic heart failure and reduced ejection fraction (PARALLELHF). Available from: https://clinicaltrials.gov/ct2/show/NCT02468232. NLM identifier: NCT02468232. Accessed August 10, 2017.

27. Kristensen SL, Martinez F, Jhund PS, et al. Geographic variations in the PARADIGM-HF heart failure trial. Eur Heart J. 2016;37(41):3167-3174.

28. Novartis. Efficacy and safety of LCZ696 compared to valsartan, on morbidity and mortality in heart failure patients with preserved ejection fraction (PARAGON-HF). Available from: http://clinicaltrials.gov/ct2/ show/NCT01920711. NLM identifier: NCT01920711. Accessed June 11, 2017.

29. Novartis. A randomized, double-blind controlled study comparing LCZ696 to medical therapy for comorbidities in HFpEF Patients (PARALLAX). Available from:http://clinicaltrials.gov/ct2/show/ NCT03066804. NLM identifier: NCT03066804. Accessed June 10, 2017.

30. Simpson J, Jhund PS, Rouleau J, et al. Effect of sacubitril/valsartan compared with enalapril, according to etiology in PARADIGM-HF. Abstract presented at: American College of Cardiology Scientific Sessions; March 2017; Washington, DC.

31. Solomon S, Packer M, Zile M, et al. The angiotensin receptor neprilysin inhibitor LCZ696 is effective across the spectrum of ejection fraction in heart failure with reduced ejection fraction. $J$ Card Fail. 2015;21(8):S45-S46.

32. Desai AS, McMurray JJ, Packer M, et al. Effect of the angiotensin receptor-neprilysin inhibitor LCZ696 compared with enalapril on mode of death in heart failure patients. Eur Heart J. 2015;36(30): 1990-1997.

33. Riddell E, Vader JM. Potential expanded indications for neprilysin inhibitors. Curr Heart Fail Rep. 2017;14(2):134-145.

34. Jong P, Yusuf S, Rousseau MF, et al. Effect of enalapril on 12-year survival and life expectancy in patients with left ventricular systolic dysfunction: a follow-up study. Lancet. 2003;361(9372):1843-1848. 
35. Pfeffer MA, Braunwald E, Moyé LA, et al. Effect of captopril on mortality and morbidity in patients with left ventricular dysfunction after myocardial infarction. N Engl J Med. 1992;327(10):669-677.

36. Dargie HJ. Effect of carvedilol on outcome after myocardial infarction in patients with left-ventricular dysfunction: the CAPRICORN randomized trial. Lancet. 2001;357(9266):1385-1390.

37. Solomon SD, Claggett B, Packer M, et al. Efficacy of sacubitril/valsartan relative to a prior decompensation: the PARADIGM-HF trial. JACC Heart Fail. 2016;4(10):816-822.

38. Novartis. Comparison Of sacubitril/valsartan enalapril on effect on ntpRo-bnp in patients stabilized from an acute heart failure episode (PIONEER-HF). Available from: http://clinicaltrials.gov/ct2/show/ NCT02554890. NLM identifier: NCT02554890. Accessed June 10, 2017.

39. Novartis. Comparison of pre- and post-discharge Initiation of LCZ696 Therapy in HFrEF patients after an acute decompensation event (TRANSITION). Available from: http://clinicaltrials.gov/ct2/show/ NCT02661217. NLM identifier: NCT02661217. Accessed June 9, 2017.

40. Novartis. Prospective ARNI vs ACE inhibitor trial to determine superiority in reducing heart failure events after MI (PARADISE-MI). Available from: http://clinicaltrials.gov/ct2/show/NCT02924727. NLM identifier: NCT02924727. Accessed June 9, 2017.

41. Maggioni AP, Anker SD, Dahlström U, et al. Are hospitalized or ambulatory patients with heart failure treated in accordance with European Society of Cardiology guidelines? Evidence from 12,440 patients of the ESC heart failure long-term registry. Eur J Heart Fail. 2013;15(10):1173-1184.

42. Novartis. UK Heart and Renal Protection (UK HARP-III). Available from: http://www.isrctn.com/ISRCTN11958993. NLM identifier: ISRCTN11958993. Accessed June 10, 2017.
43. Desai AS, Vardeny O, Claggett B, et al. Reduced risk of hyperkalemia during treatment of heart failure with mineralocorticoid receptor antagonists by use of sacubitril/valsartan compared with enalapril: a secondary analysis of the PARADIGM-HF trial. JAMA Cardiol. 2017;2(1):79-85.

44. Kristensen SL, Preiss D, Jhund PS, et al. Risk related to pre-diabetes mellitus and diabetes mellitus in heart failure with reduced ejection fraction: insights from prospective comparison of ARNI with ACEI to determine impact on global mortality and morbidity in heart failure trial. Circ Heart Fail. 2016;9(1):e002560.

45. Seferovic J, Claggett B, Seidelmann S, et al. Effect of sacubitril/valsartan versus enalapril on glycaemic control in patients with heart failure and diabetes: a post-hoc analysis from the PARADIGM-HF trial. Lancet Diabetes Endocrinol. 2017;5(5):333-340.

46. Parikh KS, Lippmann SJ, Greiner M, et al. Scope of sacubitril/valsartan eligibility after heart failure hospitalization: findings from the GWTGHF Registry (Get With The Guidelines-Heart Failure). Circulation. 2017;135(21):2077-2080

47. Perez AL, Kittipibul V, Tang WHW, et al. Patients not meeting PARADIGM-HF enrollment criteria are eligible for sacubitril/valsartan on the basis of FDA approval: the need to close the gap. JACC Heart Fail. 2017;5(6):460-463.

48. Simpson J, Benson L, Jhund PS, et al. Abstract 19155: "real world" eligibility for sacubitril/valsartan in unselected heart failure patients: data from the Swedish heart failure registry. Circulation. 2016;134:A19155.

49. Pellicori P, Urbinati A, Shah P, et al. What proportion of patients with chronic heart failure are eligible for sacubitril-valsartan? Eur J Heart Fail. 2017;19(6):768-778.

50. Zeymer U, Hochadel M, Angermann C, et al. Representativeness of the PARADIGM heart failure population in real life. Presented at: American College of Cardiology Scientific Sessions; March 2017; Washington, DC.
Vascular Health and Risk Management

\section{Publish your work in this journal}

Vascular Health and Risk Management is an international, peerreviewed journal of therapeutics and risk management, focusing on concise rapid reporting of clinical studies on the processes involved in the maintenance of vascular health; the monitoring, prevention and treatment of vascular disease and its sequelae; and the involvement of

\section{Dovepress}

metabolic disorders, particularly diabetes. This journal is indexed on PubMed Central and MedLine. The manuscript management system is completely online and includes a very quick and fair peer-review system, which is all easy to use. Visit http://www.dovepress.com/ testimonials.php to read real quotes from published authors. 